\title{
Dalla città degli amori alla città che cresce: Properzio e la Roma augustea
}

\author{
Paolo Fedeli \\ Università degli studi di Bari Aldo Moro
}

Una città può essere la somma dei nostri sentimenti o il riflesso dei nostri stati d'animo. Credo che per tutti sia così, perché una città è ben più che un luogo fisico: è la proiezione simbolica di quello che siamo o che riteniamo di essere.

Che Roma sia divenuta ben presto il riflesso dello stato d'animo di Properzio e delle sue mutevoli concezioni di poetica, lo si capisce dalla distanza che divide l'immagine della città del poeta d'amore da quella del cantore degli splendori della Roma augustea. La città del poeta d'amore è quella delle visite di notte o al sorgere del giorno in casa dell'amata $(1.3 ; 2.29 \mathrm{~b})$, delle suppliche e degli improperi alla porta chiusa perché si apra per accoglierlo (1.16), è la Roma della Suburra e delle audaci fughe di Cinzia (4.7.15-18), degli amori 'en plein air' (4.7.1920), delle meretrici che percorrono in lungo e in largo la via Sacra (2.23.15), delle scenate di gelosia nelle sordide taverne dell'Esquilino (4.8.19-20). 
Finché Properzio si considera poeta d'amore e non pratica vie diverse, Roma è per lui lo spazio dell'amore, ${ }^{1}$ come lo era stata per Catullo e, poi, per Cornelio Gallo: che al suo amore per Licoride abbia fatto da sfondo l'ambiente cittadino è Virgilio ad attestarlo, quando nella $\mathrm{X}$ bucolica per lenire le sofferenze che a Gallo provocano il tradimento e la fuga dell'amata Licoride gli suggerisce di trasferire la propria esistenza e il proprio canto d'amore in uno scenario bucolico. Che quello di Properzio sia un tipico amore cittadino lo si intuisce subito, sin da quando nella prima parte dell'elegia 1.8 egli fa di Cinzia una seconda Licoride, che vorrebbe seguire un suo occasionale ma danaroso spasimante sin nella gelida Illiria: vi rinuncerà, tuttavia, per amore del poeta e della sua poesia, e sarà quello il momento del trionfo di Properzio (1.8.31-32):

\section{illi carus ego et per me carissima Roma dicitur, et sine me dulcia regna negat.}

Per Cinzia, dunque, l'amore per Properzio s'identifica e si confonde con quello per Roma, ed è l'atteggiamento di devozione del poeta a renderle gradita la vita cittadina.

Sono gli amici stessi a ritenere l'amore della coppia elegiaca indissolubilmente legato alla città, al punto che Pontico nell'esordio dell'elegia $1,12^{2}$ può facilmente congetturare che Properzio non si decide a lasciare Roma perché la sua 'liaison' con la donna che ha scelto di cantare gli impedisce di allontanarsi da quel contesto cittadino che costituisce lo spazio dell'amore elegiaco (1.12.1-2):

$$
\begin{aligned}
& \text { quid mibi desidiae non cessas fingere crimen, } \\
& \text { quod faciat nobis, Pontice, Roma moram? }
\end{aligned}
$$

1 Riprendo qui liberamente quello che sulla città quale spazio dell'amore ho scritto in Fedeli (2010, 4-10).

2 Naturalmente se si accetta di correggere con Kraffert nel vocativo Pontice l'improbabile conscia del v. 2, che andrebbe riferito a Roma. Sulla situazione testuale cf. Fedeli (1980, 288-290). 
D'altra parte è significativo che ai periodi di dura astinenza sessuale per il tradimento faccia riscontro invariabilmente la negazione dei luoghi e dei momenti tipici della vita cittadina, tanto che, quando Properzio descrive il trionfo in amore del barbarus che gli ha sottratto la donna amata, ${ }^{3}$ la sua condizione disperata di amante abbandonato si manifesta non solo con l'inappetenza, ma anche col rifiuto di quei luoghi, come il teatro e il Campo Marzio, che dello spazio cittadino sono i simboli privilegiati (2.16.33-34):

tot iam abiere dies, cum me nec cura theatri nec tetigit Campi, nec mea mensa iuvat.

All'interno della città quale scenario dell'amore, lo spazio in cui esso si concretizza si restringe alla casa e al letto di Cinzia. Properzio non parla mai della propria casa come di un luogo d'amore, ma sempre si descrive mentre fiducioso si presenta alla porta della casa di Cinzia. Leccezione è costituita da 4.8, ma dipende dalle modalità di esecuzione del tentativo di rivalsa del poeta nei confronti del tradimento di Cinzia: se la donna amata ha deciso di scorazzare fra Roma e Lanuvio sul cocchio di un ricco ed eccentrico amante, sarà nella propria dimora a Roma, sull'Esquilino, che Properzio organizzerà un festino consolatorio con due donnine allegre. $\mathrm{Ma}$ il ritorno improvviso e inatteso di Cinzia è quello tipico di una domina che considera violato lo spazio dell'amore a lei sola riservato, e per questo motivo oltre a dettare le regole di un rinnovato foedus - provvede alle purificazioni di rito, prima di sancire la pace con una battaglia erotica sul letto di Properzio.

Se, però, la norma vuole che a Roma lo spazio degli amanti sia quello della casa di Cinzia - che anche per questo motivo palesa la condizione di meretrix più spesso di quella di matronaallora non c'è da meravigliarsi né dei frequenti accenni ai custodes

2.16.27-28 barbarus exutis agitat vestigia lumbis / et subito felix nunc mea regna tenet. 
che impediscono l'ingresso né della raffigurazione di una dimora in cui tutti possono entrare perché Cinzia si comporta come una grande cortigiana; ${ }^{4}$ una dimora dove i giovani si disputano animosamente la precedenza e con i loro reiterati appelli non le consentono di dormire. ${ }^{5}$ Se la casa di Cinzia costituisce lo spazio dell'amore, ben si capisce perché nella fase del discidium essa possa assumere una connotazione negativa, che ben s'intona con l'atteggiamento desolato e querulo di una Cinzia che soffre per la lontananza del suo uomo e per il sospetto del tradimento. In 3.6.11-18

$$
\begin{array}{ll}
\text { nec speculum strato vidisti, Lygdame lecto } & 11 \\
\text { scriniaque ad lecti clausa iacere pedes } & 14 \\
\text { ac maestam teneris vestem pendere lacertis? } & 13 \\
\text { Ornabat niveas nullane gemma manus? } & 12 \\
\text { Tristis erat domus, et tristes sua pensa ministrae } & 15 \\
\text { carpebant, medio nebat et ipsa loco, } & \\
\text { umidaque impressa siccabat lumina lana, } & \\
\text { rettulit et querulo iurgia nostra sono? }
\end{array}
$$

la condizione di abbandono e di trascuratezza in cui versa Cinzia si riflette sullo stato della sua casa: lo specchio giace abbandonato sul letto, il cofanetto con gli ingredienti per il trucco è confinato ai piedi del letto, la veste è neghittosamente gettata sulle sue spalle e nessuna pietra preziosa adorna le sue dita.

In quale quartiere di Roma il poeta collochi lo spazio dell'amore è Cinzia a dircelo, nella rievocazione di un tempo

\footnotetext{
4 2.6.1-6 Non ita complebant Ephyraeae Laidos aedis, / ad cuius iacuit Graecia tota fores; / turba Menandreae fuerat nec Thaidos olim / tanta, in qua populus lusit Ericthonius; / nec quae deletas potuit componere Thebas, / Phryne tam multis facta beata viris.

5 2.19.5-6 nulla neque ante tuas orietur rixa fenestras, / nec tibi clamatae somnus amarus erit. La rixa ante Cynthiae fenestras a cui allude Properzio nel v. 5 sarà con ogni probabilità una disputa fra spasimanti avvinazzati perché reduci dal banchetto e desiderosi di avere la precedenza nei favori sessuali, piuttosto che un alterco fra uno spasimante e il portiere o un tentativo di attirare, urlando, l'attenzione della donna.
} 
ormai lontano nel suo sfogo post mortem nei confronti dell'amante ingrato e immemore (4.7.15-18):

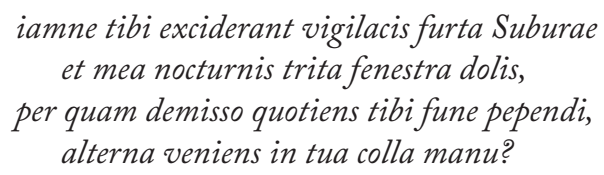

Che la scena sia ambientata nella Suburra potrà sorprendere i patetici e irriducibili sostenitori di una Cinzia matronale, ma offre una definitiva conferma a quanti ritengono che Properzio abbia voluto conferire alla donna da lui cantata i tratti di una meretrix da commedia (quanti suoi monologhi patetici, allora, piuttosto che un tono tragico potrebbero assumere cadenze paratragiche!).

Insomma, nei primi tre libri di elegie sembra proprio che abbiano un ruolo solo i luoghi convenzionali del corteggiamento e dell'amore e che nulla esista al di fuori di essi. Sembra proprio che per i monumenti della Roma augustea da parte del poeta non esista alcun interesse e si direbbe che egli passi ogni giorno accanto ad essi con lo sguardo fugace e assente. Si capisce, però, che è il genere praticato a orientare i contenuti della poesia. Talora, però, possono presentarsi esigenze diverse, tali da preannunziare quel mutamento di rotta che nel IV libro diverrà esplicito.

Ha scritto Paul Zanker che "l'immagine complessiva di una città in una particolare situazione storica «rappresenta un coerente sistema di comunicazione visiva, in grado di influenzare gli abitanti anche a livello inconscio per il fatto stesso della sua continua presenza."

Neppure il poeta d'amore può restare insensibile di fronte al fascino della città che cresce: con i suoi interventi nei campi più diversi, Augusto è intento a riscrivere tutto e, al tempo stesso,

$6 \quad$ Zanker (1989, 23). 
"iscrive se stesso in ogni aspetto della vita, pubblica e privata." ${ }^{\prime} \mathrm{E}$ l'architettura, in particolare, ad avere un ruolo di primario rilievo nel suo progetto di conquista di un generale consenso, grazie alla felice intuizione di legare il programma di rinnovamento edilizio a quello di esaltazione dei valori religiosi: già a ridosso di Azio egli da un lato favorisce la costruzione sul Palatino dell'imponente tempio di Apollo, dall'altro erige il Mausoleo nella parte settentrionale del Campo Marzio e trasforma profondamente il Foro. Cassio Dione (52.30.1) attribuisce a Mecenate il merito di aver suggerito a Ottaviano, nel 29 a.C., il necessario abbellimento della città quale punto di primaria importanza ai fini della conquista del consensus. Da allora gli interventi edilizi si susseguirono con un ritmo incalzante e furono tali da incidere sensibilmente sulla fisionomia della città repubblicana. ${ }^{8}$ Come attesta Svetonio, ${ }^{9}$ dando prova di accortezza e lungimiranza Augusto si preoccupò di coinvolgere in qualità di committenti i personaggi di maggior rilievo, sicché nel corso degli anni «membri della famiglia imperiale, vecchi alleati politici, seguaci di Antonio poi passati dalla sua parte, famiglie cooperanti della nobiltà tradizionale, membri dei nuovi ceti in ascesa, e, non da ultimo, senato e popolo; tutti furono chiamati a collaborare, in una grande e concertata azione di consenso». ${ }^{10}$

7 Barchiesi (1994, 59).

8 Un ottimo sguardo d'insieme, oltreché in Zanker (2013, 51-56), in Sommella - Migliorati $(1991,291-7)$ e soprattutto in Favro 1996, in particolare nelle pgg. 79-142.

9 Suet. Aug. 29.4-5 sed et ceteros principes viros saepe hortatus est ut pro facultate quisque monimentis vel novis vel refectis et excultis urbem adornarent. Multaque a multis tunc extructa sunt, sicut a Marcio Philippo aedes Herculis Musarum, a L. Cornificio aedes Dianae, ab Asinio Pollione atrium Libertatis, a Munatio Planco aedes Saturni, a Cornelio Balbo theatrum, a Statilio Tauro amphitheatrum, a M. vero Agrippa complura et egregia.

10 Così Hölscher $(2009,151)$; sulla partecipazione delle gentes cfr. anche Sommella - Migliorati (1991, 291-5). 
L'elegiaco Properzio sin dal II libro sembra preannunciare gli esiti dichiaratamente augustei del IV, pur evitando - almeno per ora - di entrare in aperto conflitto con i contenuti della poesia erotica. Sono gli anni in cui Augusto dà inizio all'opera di restauro e di nuova edificazione dei templi e degli edifici pubblici: testimone attento e interessato dei mutamenti della città, il cantore di Cinzia capisce bene che l'introduzione nella poesia erotica di tematiche connesse con l'attività edilizia rischia di risultare piuttosto stravagante in un canzoniere per la donna amata, e ricorre, quindi, a un ingegnoso espediente: invece di accogliere all'interno del tessuto erotico elementi ad esso estranei, presenterà come accessorio proprio l'argomento erotico. È quello che egli fa nella XXXI elegia del II libro, dove il tenue legame con la poesia di corteggiamento galante è definito nel distico iniziale (2.31.1-2):

\section{Quaeris cur veniam tibi tardior? Aurea Phoebi Porticus a magno Caesare aperta fuit.}

A un appuntamento con l'amata, Properzio non è giunto in orario; colpevole del ritardo che Cinzia gli rimprovera è stata l'inaugurazione del portico di Apollo, annesso al tempio del dio sul Palatino, di cui egli dà una parziale descrizione. Nel marasma dell'attuale II libro, confluenza a parer mio pressoché certa di due libri originari, ${ }^{11}$ l'elegia ha tutta l'aria di esserci giunta in modo incompleto; tuttavia il suo probabile stato frammentario non c'impedisce di cogliere il forte impatto ideologico del complesso. ${ }^{12}$ Edificato su un terreno di proprietà di Ottaviano che era stato colpito dal fulmine, ${ }^{13}$ il tempio era stato votato nel 36 a.C. dopo la vittoria su Sesto Pompeo a Nauloco ${ }^{14}$ e dedicato

11 Ne ho discusso ampiamente nell'introduzione al mio commento del II libro Fedeli $(2005,21-35)$.

12 Sul tempio di Apollo come sintesi di un progetto politico-culturale cf. Zanker $(1989,97)$.

13 Cf. Suet. Aug. 29.3; Cass.Dio 49.15.5.

14 Vell. 2.81.3; Cass.Dio 49.15.5. 
nel 28 a.C..$^{15}$ poiché la casa di Augusto sul Palatino era collegata alla terrazza del tempio da una rampa di accesso, dimora del principe e luogo di culto del dio suo protettore costituivano un complesso unitario di grande significato ideologico. ${ }^{16}$

Il fatto stesso di aver inserito in una raccolta di poesia d'amore un carme che, invece, tesse l'elogio di una simile realizzazione del principe costituisce di per sé una significativa testimonianza di adesione del poeta elegiaco al programma edilizio del magnus Caesar (v. 2): basterebbe questo esempio a far capire come sia fuori luogo tacciare il poeta di totale disinteresse nei confronti del progetto edilizio di Augusto. ${ }^{17}$ Non solo la XXXI elegia del II libro, ma anche i vv. 11-16 della successiva, con la loro presentazione della porticus di Pompeo ci mostrano un poeta che, sensibile all'aspetto della città e pienamente partecipe delle sue trasformazioni, descrive con compiaciuta insistenza i luoghi in cui si svolge la vita dei giovani, dal Campo Marzio ai portici e ai templi.

Si può dire che proprio l'elegiaco Properzio sia divenuto il più convinto cantore del progressivo mutamento della fisionomia della città dei tempi suoi: come Augusto aveva ben capito «che l'architettura, intesa come strumento per dare una nuova forma a Roma, era un mezzo tanto efficace quanto la poesia», ${ }^{18}$ così Properzio, una volta ammesso nella cerchia di Mecenate, relegati nel I libro e ormai dimenticati gli eccidi del bellum Perusinum compiuti da Ottaviano al tempo della presa di Perugia, si era reso ben conto dell'importanza e dell'originalità che avrebbe potuto conseguire la sua poesia elegiaca in quanto fiancheggiatrice del nuovo modo di concepire e di realizzare la città.

15 Cass.Dio 53.1.3; CIL I ${ }^{2}$ 214.245.249.

16 Cf. Zanker (1989, 57); (2014, 221-244) e, per il forte effetto scenografico, Sauron $(2014,85)$.

17 È questo il giudizio espresso da Zanker $(2014,222)$.

18 Wallace-Hadrill $(2014,137)$. 
Quando, fra il 20 e il 15 a.C., egli compone il IV libro, la sua adesione al programma di Augusto appare sincera e convinta sin dalla prima, programmatica elegia e per lui si tratta solo di saper scegliere, nella presentazione di una raccolta a metà strada fra poesia delle origini e poesia d'amore, quale aspetto celebrare dell'attività del principe: non a caso egli decide di privilegiare il restauro di edifici sacri ormai fatiscenti e la nuova costruzione di templi e di teatri. Illuminante è l'esplicita dichiarazione di poetica che sovrintende a tale scelta: il poeta, infatti, si considera in procinto d'intraprendere un'impresa che non ha nulla da invidiare a quella di un fondatore o rifondatore di città: quando, infatti, nel v. 57 della 4.1 mette in chiaro l'aspirazione a moenia disponere versu, egli fa di sé un singolare fondatore, che si serve della poesia in luogo dell'aratro. C'è in quell'espressione la consapevolezza che, celebrando la crescita delle mura e della città, l'attività del poeta finisca per coincidere con quella di un Anfione, che al suono della lira edificò le mura di Tebe. ${ }^{19}$ Sullo stesso piano dell'attività edilizia promossa da Augusto, grazie alla quale Roma sta mutando la sua fisionomia, si colloca dunque quella del poeta, che muta il suo modo di far poesia adattandolo alle istanze dei tempi nuovi; e come Augusto con la sua opera di restauro e di nuova costruzione di templi e di edifici pubblici sta rifondando Roma, così Properzio si accinge a riscrivere Roma con la sua poesia delle origini dei sacra, degli dei e dei cognomina locorum. Ma come Roma cresce nel rispetto della continuità col passato, così il poeta apre nuove vie alla poesia elegiaca senza escludere quelle già percorse nel passato. ${ }^{20}$

19 Osserva Gazich $(1997,324)$ che mentre moenia "può riferirsi a materiali riguardanti la fondazione e la storia delle origini, moenia disponere non segnala solo la tensione tra materia gravis e arte tenuis (...), ma definisce il modo in cui questa materia, prelevata da contesti epici, viene introdotta in ambito elegiaco, cioè ricodificata attraverso una ridistribuzione degli elementi e un loro reciproco adattamento".

20 Il motivo del poeta-architetto e fondatore verrà ripreso nel v. 67 (Roma, fave, tibi surgit opus!), dove il verbo surgere non è in rapporto soltanto col libro di poesia di Properzio, ma per estensione di significato con la città interà: alla città che 
Nell'accingersi ad aprire nuove vie alla sua poesia, Properzio guarda a Virgilio, in particolare alla 'passeggiata' di Evandro e di Enea nei luoghi in cui sorgerà Roma (Aen. 8.307368), dall'Ara Massima al Foro Boario sino al Palatino, dov'è la dimora di Evandro: la forza del modello è tale che nell'elegia incipitaria del IV libro sarà il poeta stesso ad appropriarsi del ruolo di Evandro e ad indicare a uno straniero le realizzazioni più significative della maxima Roma augustea, a cominciare dall'edilizia templare. Anche in questo a indicargli la via era stato Virgilio, che nel descrivere il triplice trionfo del 29 a.C. effigiato sullo scudo di Enea aveva insistito sul momento religioso, con la consacrazione di un numero infinito di templi agli dèi italici da parte di Augusto (Aen. 8.714-6 at Caesar, triplici invectus Romana triumpho / moenia, dis Italis, votum immortale, sacrabat I maxima tercentum totam delubra per urbem).

Quando il poeta elegiaco, forte di un simile precedente, in apertura della prima elegia offre allo sguardo dell'bospes la visione degli aurea templa che si ergono nella loro maestosa imponenza, non si propone soltanto di esaltarne lo splendore e l'architettonica perfezione, ma vuole mettere in risalto la pietas del principe nei confronti degli dèi e la fusione della nuova Roma augustea col momento religioso. Lo sguardo del poeta indugia sul tempio di Apollo e sugli altri del Palatino, prima di soffermarsi su quello di Giove Capitolino (vv. 7-8). Al Palatino fa ritorno con la casa Romuli recentemente restaurata (v. 9) per passare poi alla Curia (vv. 11-12) e, quindi, di nuovo al Campidoglio (vv. 13-14), prima di rivolgere lo sguardo ai teatri (vv. 15-16): l'andamento desultorio e segnato da continui ritorni sui propri passi non dà l'impressione di un'ordinata e composta periegesi,

cresce corrisponde il libro di poesia che cresce, sino a raggiungere il suo aspetto definitivo. Lo stesso motivo verrà riproposto da Ovidio, ironicamente negli Amores (1.1.27 sex mibi surgit opus numeris, in quinque residat), seriamente nei Fasti (4.830 auspicibus vobis hoc mibi surgat opus) e nei Tristia (2.559-560 surgens ab origine mundi / in tua deduxi tempora, Caesar, opus). 
come quella di Evandro e di Enea nell'VIII libro della virgiliana Eneide, ma vuole riprodurre il soffermarsi dello sguardo sugli edifici che più lo colpiscono. Si capisce, tuttavia, che punto d'osservazione privilegiato resta il Palatino, ideale per instaurare un confronto tra la Roma del passato e quella del presente e per legare strettamente luoghi di culto (il tempio di Apollo, divinità protettrice di Augusto) e luoghi del potere (in primo luogo la dimora del principe).

Quella dei versi iniziali di 4.1 è solo una prima presentazione: il tempio di Apollo Palatino verrà riproposto nell'elegia che celebra il XV anniversario di Azio (4.6), quello di Giove Capitolino nell'elegia di Tarpea (4.4), e nel corso del libro altri templi saranno ricordati e celebrati: nella $X$ elegia quello di Giove Feretrio, che versava in uno stato d'abbandono e d'incuria tali da giustificarne l'inclusione da parte di Augusto fra i templi di nuova costruzione, ${ }^{21}$ laddove sia Cornelio Nepote sia Livio parlano di restauro. ${ }^{22}$ Nella IX elegia, oltre all'Ara Massima in onore di Ercole trova un implicito riconoscimento l'attività in campo edilizio dell'augusta imperatrice: il ruolo insolitamente ampio e importante lì accordato al culto della Bona Dea e al luogo sacro in cui viene celebrato si giustifica se si considera che, mentre il programma edilizio di Augusto era in una fase d'intenso sviluppo, Livia decise di affiancarlo promuovendo proprio il restauro del tempio della Bona Dea Subsaxana, sul fianco orientale dell'Aventino: lo attesta Ovidio, che nel V libro dei Fasti non si limita a parlare del tempio e della sua origine, ma ricorda l'efficace e decisiva opera di restauro promossa da Livia (vv. 149-158): ${ }^{23}$

\footnotetext{
Res gest. 19.2 aedes in Capitolio Iovis Feretri ...feci.

22 Nep. Att. 20.3; Liv. 1.10.6; cfr. Coarelli (1996, 135-6).

23 Anche se dell'epoca del restauro mancano notizie certe e neppure Augusto ne parla nel cap. 19 delle sue Res gestae riservato ai templi restaurati o edificati, non è da escludere che esso sia stato realizzato durante il periodo della sua assenza da
} 
est moles nativa loco, res nomina fecit: appellant Saxum; pars bona montis ea est.

Huic Remus institerat frustra, quo tempore fratri prima Palatinae signa dedistis aves.

Templa Patres illic oculos exosa viriles leniter acclini constituere iugo.

Dedicat haec veteris Clausorum nominis heres, virgineo nullum corpore passa virum.

Livia restituit, ne non imitata maritum esset et ex omni parte secuta suum.

Vitruvio, rivolgendosi ad Augusto nella prefazione del De architectura, confessa di non aver osato pubblicare le sue riflessioni finché il principe era impegnato nella lotta politica e militare (1 praef. 1$)$, temendo di disturbarlo in un momento poco opportuno. "Quando però - egli continua - notai che tu non ti prendevi cura soltanto della vita pubblica della comunità e dell'organizzazione dello stato, ma anche dell'opportunità di dare sviluppo all'edilizia pubblica, in modo che per opera tua non solo lo stato risultasse accresciuto grazie alle nuove province, ma la grandezza del potere si manifestasse anche nello straordinario prestigio degli edifici pubblici, ritenni di non dover lasciare passare la prima occasione per pubblicare, dedicandoli a te, quei miei scritti sull'argomento in questione". ${ }^{24} \mathrm{E}$, subito dopo, così ribadisce e completa il suo pensiero: "Cominciai a comporre quest'opera dedicata a te, perché mi accorsi che tu avevi fatto costruire e continuavi a far costruire molti edifici, e che anche nel tempo a venire avresti curato che gli edifici pubblici e privati fossero degni di essere affidati alla memoria dei posteri, in rapporto alla grandezza delle tue imprese" (1 praef. 3 ).

Non a caso, nel contesto properziano, dagli aurea templa lo sguardo si sposta ben presto (vv.11-14) sulla Curia e sui senatori antichi e contemporanei: il senato è il simbolo della persistenza

Roma fra il 22 e il 19 a.C.: cf. Fox (1996, 170), Spencer (2001, 273 n. 28), Welch (2004, 68-72) e sul tempio Chioffi (1993, 200-1).

24 Vitr. 1 praef. 2; le traduzioni del De architectura sono quelle della Romano 1997. 
dei valori repubblicani, e ben si capisce che, nel tessere l'elogio del programma edilizio di Augusto, il poeta abbia inteso mettere in luce non solo il suo rispetto dei valori religiosi (l'architettura sacrale), ma anche la continuità fra ideologia imperiale e antichi valori repubblicani. Considerata, poi, nell'ambito delle realizzazioni edilizie, la Curia Iulia segna la continuità col programma di Cesare, che l'aveva voluta per rimpiazzare la Curia Hostilia: cominciati nel 44 a.C., i lavori erano stati fortemente osteggiati dall'aristocrazia senatoria ${ }^{25}$ e non vennero completati prima del 29 a.C., quando la nuova Curia fu inaugurata da Ottaviano. ${ }^{26}$

Tra gli edifici pubblici i teatri hanno una funzione di primaria importanza perché, oltre ad essere il luogo d'incontro privilegiato del principe col popolo, svolgono una significativa funzione culturale: se Roma voleva assumere l'aspetto di una capitale ellenistica, era necessario che si dotasse di grandi e splendidi teatri, in grado di esercitare la stessa funzione svolta, in particolare, da Atene. ${ }^{27}$ Nella properziana 4.1 ai teatri è riservato un solo distico (vv. 15-16), in cui vengono messe in risalto la modernità dell'uso dei vela e la raffinata consuetudine di profumare la scena. Su questo terreno il confronto tra l'epoca augustea e il tempo antico si rivela impietoso: a sollecitarlo saranno state sia la ricostruzione del teatro di Pompeo, voluta da Ottaviano ancor prima di Azio, nel 32 a.C. ${ }^{28}$ sia l'edificazione del teatro di Marcello, che era certamente attivo nel 17 a.C.; ${ }^{29}$ per quello che possiamo desumere dalle elegie databili del IV libro, non era stato ancora completato il teatro di Balbo, inaugurato poi nel 13 a.C. ${ }^{30}$ Nell'ambito della riorganizzazione augustea dello spazio urbano s'inserirà, nei versi successivi, l'implicita con-

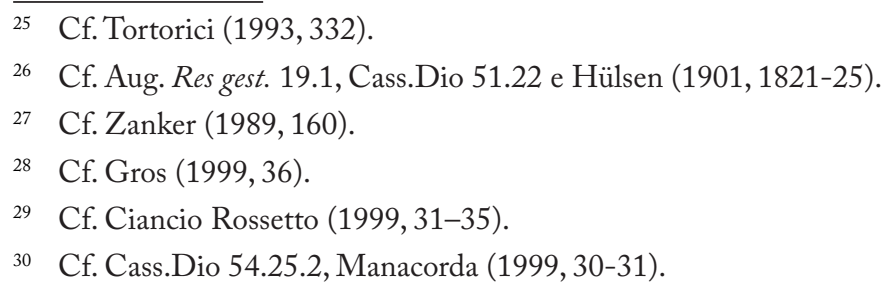


trapposizione dello spazio della festa dei tempi antichi a quello della Roma augustea (vv. 21-22 Vestalia, vv. 23-24 Compitalia, vv. 25-26 Lupercalia). ${ }^{31}$

Quando nel 19 a.C. Augusto rientrò a Roma dopo tre anni di assenza, Properzio pensò bene di celebrare il suo trionfale ritorno dalle vittoriose campagne di guerra istituendo un parallelo nella IX elegia col leggendario transito nel Lazio di Ercole reduce dalla Spagna dopo l'esito felice della decima fatica. Fondandosi sulla testimonianza di Augusto nelle sue Res gestae (cap. 11), Stephen Harrison ha ricostruito il suo percorso da porta Capena sino al Foro e al Campidoglio e ha constatato che esso include proprio i luoghi della IX elegia del IV libro, dal tempio della Bona Dea Subsaxana al Velabro, all'Ara Massima e alle pendici del Palatino. ${ }^{32}$

Ma c'è di più: secondo una felice intuizione della Fantham, ${ }^{33}$ ripresa da Labate, «la struttura del IV libro properziano sembra prevedere che il poeta antiquario visiti con le sue illustrazioni e le sue ricostruzioni eziologiche i luoghi di quella stessa area del centro di Roma che lo sguardo proemiale aveva panoramicamente abbracciato». ${ }^{34}$ Della I e della IX elegia si è già detto: tuttavia non è soltanto la serie di carmi eziologici, ma il libro nel suo complesso, che dà l'impressione di organizzarsi in modo da riprodurre l'aspetto della città. Fatta eccezione per l'XI elegia, il cui scenario è costituito dall'oltretomba, tutte le altre sono funzionali all'illustrazione della città: assolvono questo compito, nella II elegia la statua di Vertumno nel Foro (vv.3-6), il Tevere col suo corso mutato (vv. 7-10) e il vicus Tuscus

\footnotetext{
31 Sulla riorganizzazione augustea dello spazio urbano e in particolare sui Compitalia cfr. Fraschetti (2005, 184-242).

32 Harrison (2005, 118-120); egli, inoltre, ha formulato l'ipotesi che Properzio si sia servito dell'aition della fondazione dell'Ara Massima per ricordare la fondazione recente dell'Ara Fortunae reducis.

33 Fantham (1997, 128. 131); (2009, 65).

34 Labate (2010, 158 n.1).
} 
(vv. 49-50); nella III, i sacella, i compita (v. 57), la porta Capena fra il Celio e l'Aventino (vv. 71-72); nella IV, il Tarpeium saxum (v. 1), il piccolo tempio di Giove Feretrio (v. 2), il Campidoglio (vv. 27 e 93-94) e il tempio di Giove Capitolino (v. 30), il Foro (vv. 11-12), la Curia (v. 13), lo spazio urbano e suburbano destinato alla celebrazione dei Parilia (vv. 73-78), il tempio di Vesta (vv. 17-18; 45-46); nella V, porta Collina (v. 11) e i sepolcreti del Campus Sceleratus; nella VI, il tempio di Apollo sul Palatino (v. 11); nella VII, la Suburra (v. 15); nell'VIII, l'Esquilino e le fonti sulle sue pendici (v. 1), gli horti di Mecenate (v. 2), il tempio di Diana sull'Aventino (v. 29), l'asylum (la depressione fra il Campidoglio e la rocca capitolina, nel v. 30), la porticus di Pompeo (v. 75), il Foro e i teatri (v. 77); nella X, il tempio di Giove Feretrio sul Campidoglio.

È ben noto che la politica di Augusto sulla città, pur abbandonando la grandiosità dei progetti di Cesare, non volle rappresentare un momento di rottura nei confronti del suo programma. Dell'edilizia templare Augusto stesso nelle Res gestae tenderà a mettere in luce gli interventi di risanamento e di restauro che avevano caratterizzato gli inizi, sostanzialmente conservativi, della sua attività (20.4 duo et octoginta templa deum in urbe consul sextum ex auctoritate senatus refeci, nullo praetermisso quod eo tempore refici debebat). ${ }^{35}$

Alla scelta augustea della continuità piuttosto che della rottura fa riscontro un analogo atteggiamento del poeta architetto. Nel discorso di Properzio all'hospes nella prima parte dell'elegia incipitaria del IV libro, a prima vista si ha l'impressione che sia privilegiato il motivo del contrasto e che, per di più, esso si manifesti nei campi più diversi: il più evidente, quello

35 Sul progetto di Cesare, sulle resistenze del senato repubblicano e sul declino dei templi e dei luoghi di culto cfr. Zanker (1989, 24-9), Sommella - Migliorati (1991, 287-91); sulle fasi di passaggio dalla Roma cesariana a quella augustea cf. ora La Rocca $(2014,93-5)$. 
fra presente e passato, prende le mosse dall'invito all'hospes (vv. 1-4) perché s'immagini un solitario paesaggio di colli e di campi erbosi, là dove ora si erge imponente la Roma di Augusto, e un luogo di pascolo per le sfinite giovenche di Evandro là dove ora splendido s'innalza il tempio di Apollo sul Palatino. Tuttavia, come qui e nei confronti istituiti nel contesto successivo non c'è condanna del lusso e degli splendori della Roma di Augusto, così non c'è neppure rifiuto del passato, non solo perché esso s'identifica con le origini della città, ma anche perché tutto è considerato alla luce di una ininterrotta continuità fra la Roma di un tempo e quella di Augusto.

Il senso della continuità - che anche nel programma politico Augusto aveva preferito alla rottura nei confronti del passato, con la sua concezione dell'impero inteso come una prosecuzione della repubblica - nell'elegia incipitaria si riflette nella raffigurazione del principe che innova nel solco della tradizione. Anche per questo motivo il confronto tra passato e presente viene sempre inteso come un fenomeno di crescita ed è questo il principio che governa il IV libro delle elegie di Properzio. Cornelia, protagonista dell'ultima elegia, col suo discorso di fronte al tribunale degli Inferi costituisce una realizzazione perfetta di una tale concezione della storia di Roma: da un lato Cornelia è orgogliosamente legata al suo passato familiare, che s'identifica con la gloria degli Scipioni e con i momenti più significativi della storia di Roma; dall'altro, però, nelle parole rivolte ai figli mostra di concepire il rapporto col passato come un fenomeno di crescita, che può solo preludere a un futuro migliore per la stessa Roma. Il poeta, per parte sua, lo aveva messo in chiaro fin dall'inizio della prima elegia del IV libro, quando nell'istituire il confronto tra i fictiles dei dei tempi antichi e gli aurea templa del presente augusteo (v. 5 fictilibus crevere deis haec aurea templa) si era servito proprio del verbo crescere, che tra il passato e il presente stabilisce un solido nesso e dà il senso di un presente che si alimenta del passato nell'intento di perfezionarlo e di farne una cosa nuova. 
Con Ovidio, che pure è sensibile all'influsso del più anziano Properzio, muta radicalmente la funzione della città augustea nel libro di poesia d'amore. ${ }^{36}$ Nell'Ars i luoghi della Roma augustea si limitano a costituire lo sfondo necessario per $\mathrm{i}$ precetti in materia amorosa del poeta: nel I libro, in particolare, essi s'identificano con gli ambienti più adatti agli incontri, ai corteggiamenti, alle conquiste d'amore. In assenza di celebrazioni sia dell'edilizia templare sia di quella pubblica, paradossalmente la città augustea sembra concepita proprio per favorire il corteggiamento e gli approcci: in tal modo essa diviene parte attiva dellopera di conquista amorosa.

Insieme al ruolo della città augustea ora muta, nel libro di poesia elegiaca d'amore, il modo stesso di concepire il rapporto col passato: il senso augusteo della continuità, che Properzio aveva fatto suo, viene sostituito dall'aperta rottura col passato, in nome di un atteggiamento dichiaratamente modernista, che conduce a una decisa e convinta svalutazione di tutto ciò che al passato appartiene. ${ }^{37} \mathrm{Il}$ testo chiave per capire come il punto di vista di Ovidio sia antitetico a quello di Properzio è costituito da Ars 3.113-128:

$$
\begin{aligned}
& \text { simplicitas rudis ante fuit; nunc aurea Roma est } \\
& \text { et domiti magnas possidet orbis opes. } \\
& \text { Aspice quae nunc sunt Capitolia quaeque fuerunt: } \\
& \text { alterius dices illa fuisse Iovis. } \\
& \text { Curia consilio nunc est dignissima tanto, } \\
& \text { de stipula Tatio regna tenente fuit; } \\
& \text { quae nunc sub Phoebo ducibusque Palatia fulgent, } \\
& \text { quid nisi araturis pascua bubus erant? } \\
& \text { Prisca iuvent alios, ego me nunc denique natum } \\
& \text { gratulor: baec aetas moribus apta meis, } \\
& \text { non quia nunc terrae lentum subducitur aurum } \\
& \text { lectaque diverso litore concha venit, }
\end{aligned}
$$

\footnotetext{
36 Oltre al libro della Piastri (2004) resta fondamentale Labate (1984, 48-64. 81-85).

37 Buone osservazioni in Piastri $(2004,82)$.
} 


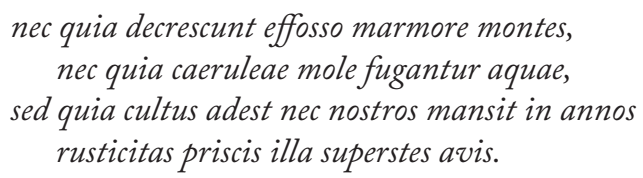

Lo schema è sempre quello della 'passeggiata archeologica'; ma mentre in Virgilio e poi in Properzio i luoghi del passato servivano a caratterizzare, ma senza svilirla, la rudis simplicitas dei tempi antichi, in Ovidio la Roma augustea non è aurea per lo splendore dei suoi templi, ma perché possiede ricchezze immense, frutto delle guerre di conquista (vv. 113-4). Assumono, allora, un senso diverso i luoghi e gli edifici che segnano la storia della città: anche qui, come in Properzio, compaiono il Campidoglio, la Curia, il tempio di Apollo sul Palatino; ma essi servono solo a fissare la distanza che inesorabilmente divide il passato dal presente, e del passato sanciscono l'indiscussa inferiorità. A confronto di quello che era in passato, il Campidoglio augusteo sembra dedicato a un Giove diverso; la Curia, che ora accoglie nel modo più degno i senatori, al tempo di Tazio era fatta di paglia; il Palatino, che ora risplende per il tempio di Apollo, nella Roma delle origini era un pascolo per i buoi. Per parte sua il poeta non può che proclamare la propria felicità perché ha avuto la fortuna di nascere nel presente, caratterizzato dall'opulenza e dalla magnificenza.

Lo stesso schema resiste anche nei Fasti, benché l'immagine della Roma arcaica sia considerata alla luce della grandezza futura: quando, però, si tratta d'instaurare un confronto con gli splendori del presente augusteo, il passato è simbolo di una semplicità che sconfina nella rozzezza. Emblematica è la presentazione della Roma delle origini nel I libro, aperta da un distico che contrappone alla povertà del passato le ricchezze del presente (Fast. 1,197-198):

pluris opes nunc sunt quam prisci temporis annis, dum populus pauper, dum nova Roma fuit. 
Ovidio ha occhi solo per la Roma augustea: di essa esalta l'oro dei templi e lo splendore degli edifici, ${ }^{38}$ e aureus è l'epiteto che assegna al Campidoglio (Fast. 6.73).

Tutto ciò appartiene al tempo felice della produzione ovidiana. Quando, però, il poeta cade in disgrazia e viene relegato nella solitudine remota di Tomi, nella poesia dell'esilio prendono a convivere due volti della Roma augustea, quello ufficiale e quello ideale, con l'inevitabile conseguenza della nostalgia della città perduta. Col trascorrere del tempo nel lontano luogo di relegazione l'immagine di Roma serve a dare concretezza e drammatico spessore al contrasto fra la vita della capitale e quella dell'inospitale Tomi, così diversa dalla pulcherrima Roma augustea (Pont. 1.2.81).

Nonostante le suppliche, Ovidio sa bene in cuor suo che mai potrà fare ritorno a Roma per l'inflessibile decisione del principe. Sarà il libro, allora, a recarsi a Roma al posto suo e a salutare i luoghi a lui più cari: il poeta lo aveva previsto sin dall'esordio del I libro dei Tristia, ${ }^{39}$ ma è solo nell'elegia proemiale del III che il libro giunge a Roma e, inatteso, si presenta ai lettori e li prega di accoglierlo con animo amico. A loro chiede d'indicargli quella che dovrà essere la meta del suo percorso, e si capisce bene che sta pensando a una delle biblioteche pubbliche della città augustea: fra i lettori ovidiani, però, solo uno è disposto a mostrargli la strada e con lui il libro compie lo stesso cammino che nell'VIII dell'Eneide Enea aveva percorso con Evandro. Sarà il suo occasionale accompagnatore a indicargli i monumenti della città che cresce, lungo un percorso che ha inizio nei Fori di Cesare e di Augusto e si snoda lungo la via Sacra sino al tempio di Vesta, dove un tempo sorgeva la reggia di Numa, e poi, girando a destra per la porta Palatina,

\footnotetext{
38 Fast. 1.77-78 flamma nitore suo templorum verberat aurum / et tremulum summa spargit in aede iubar.

39 Trist. 1.1.1-16.
} 
sino al tempio di Giove Statore. Affascinato da tanti e tali mirabilia, il libro non riesce a trattenere lo stupore quando ai suoi occhi si presenta il palazzo di Augusto $;^{40}$ di lì, percorrendo un'alta gradinata, raggiunge il tempio di Apollo Palatino e, dopo aver ammirato il portico delle Danaidi, invano cerca di essere accolto nella biblioteca del tempio, prima, in quella situata in prossimità del teatro di Marcello, poi, infine in quella annessa all'atrium Libertatis. La città che ai tempi dell'Ars aveva aperto le vie, i teatri, i monumenti al maestro di avventure galanti, la città tanto celebrata nei suoi versi ora lo ripaga, ingrata, dapprima con l'indifferenza, poi con l'aperta ostilità nei confronti del suo libro. Al poeta elegiaco, rassegnato alla sua condizione di esule, resta solo la possibilità d'immaginarsi l'ormai perduta Roma di Augusto, e di riviverla nel ricordo.

\section{Bibliografia}

Barchiesi, A. 1994. Il poeta e il principe. Ovidio e il discorso augusteo, Roma - Bari.

Chioff, L. 1993. Bona Dea Subsaxana, LTVR I: 200-201.

Ciancio Rossetto, P. 1999. Theatrum Marcelli, LTVR V: 31-35.

Coarelli, F. 1996. Iuppiter Feretrius, Aedes, LTVR III: 135-136.

Fantham, E. 1997. Images of the City: Propertius' Nerw-old Rome. In: The Roman Cultural Revolution, ed. by T. Habinek-A. Schiesaro, Cambridge: $122-135$.

2009. Latin Poets and Italian Gods, Toronto - Buffalo - London.

Favro, D. 1996. The Urban Image of Augustan Rome, Cambridge.

40 Trist. 3.1.35 Iovis haec, dixi, domus est?. Lo stupore sarà stato provocato dal pensiero che lì abita il rappresentante di Giove in terra, piuttosto che dall'aspetto, almeno a quanto attesta Suet. Aug. 72.1 habitavit primo iuxta Romanum forum supra Scalas anularias, in domo quae Calvi oratoris fuerat: postea in Palatio, sed nibilo minus aedibus modicis Hortensianis, et neque laxitate neque cultu conspicuis, ut in quibus porticus breves essent Albanarum columnarum, et sine marmore ullo aut insigni pavimento conclavia. 
Fedeli, P. 1980. Properzio. Elegie. Libro I, introduzione, testo critico e commento, Firenze.

Cambridge.

2005. Properzio. Elegie. Libro II, introduzione, testo e commento,

2010. Properzio: Lo spazio dell'amore. In: R. Cristofoli, C. Santini, F. Santucci (eds.), Tempo e spazio nella poesia di Properzio, Assisi: 4-26.

Fox, M. 1996. Roman Historical Myths. The Regal Period in Augustan Literature, Oxford.

Fraschetti, A. 2005. Roma e il principe, Roma - Bari.

Gazich, R.1997. 'Moenia disponere': Properzio, Callimaco e la città augustea, Aevum(ant), 10: 289-336.

Gros, P. 1999. Theatrum Pompei, LTVR V: 36.

Harrison, S. J. 2005. Hercules and Augustus in Propertius 4.9, PLLS 12: 117-131.

Hölscher, T. 2009. Potere, spazio ed effetti visivi: le apparizioni degli imperatori nell'architettura romana In: Resistenza del Classico, a cura di R. Andreotti, Milano: 137-59.

Hülsen, C. 1901. Curia, RE, IV 2: 1821-1825.

Labate, M. 1984. L'arte di farsi amare, Pisa.

.2010. Passato remoto. Età mitiche e identità augustea in Ovidio, Pisa - Roma.

La Rocca, E. 2014. La Roma di mattoni diventa marmo, in Augusto, catalogo della mostra (Roma 2014), a cura di E. La Rocca, C. Parisi Presicce, A. Lo Monaco, C. Giroire, D. Roger, Milano: 92-105.

Manacorda, D. 1999. Theatrum Balbi, LTVR V: 30-31.

Piastri, R. 2004. L'elegia della città. Roma nella poesia elegiaca di Ovidio, Vercelli.

Romano, E.1997. Vitruvio. 'De architectura', a cura di P. Gros, introduzione e commento di A. Corso e E. Romano, Torino.

Sauron, G. 2014. Mito e potere: la mistificazione augustea. In: Augusto, catalogo della mostra (Roma 2014), a cura di E. La Rocca, C. Parisi Presicce, A. Lo Monaco, C. Giroire, D. Roger, Milano: 85-88.

Sommella, P. - Migliorati 1991: P. Sommella, P.; Migliorati, L. (1991). Il segno urbano, in AA.VV., Storia di Roma, II 2, Torino: 287-309. 
Spencer, D. 2001. Propertius, Hercules, and the Dynamics of Roman Mythic Space in Elegy 4.9, Arethusa 34: 259-84.

Tortorici, E. 1993. Curia Iulia, LTVR I: 332.

Wallace-Hadrill, A. 2014. Ottaviano e il tramonto della repubblica romana, in Augusto, catalogo della mostra (Roma 2014), a cura di E. La Rocca, C. Parisi Presicce, A. Lo Monaco, C. Giroire, D. Roger, Milano: 134-7. Welch, T. S. 2004. Masculinity and Monuments in Propertius 4.9, AJPh 125: 61-90.

Zanker, P. 1989. Augusto e il potere delle immagini, trad. ital., Torino. .2013. La città romana, Roma - Bari.

2014. Il tempio di Apollo Palatino in Properzio e i suoi resti. In:Properzio e l'età augustea. Cultura, storia, arte, a cura di G. Bonamente, R, Cristofoli, C. Santini, Turnhout: 221-44. 